\title{
Revista \\ Barreiras no rastreamento do câncer de mama e o papel da enfermagem: revisão integrativa
}

\author{
Barriers in the breast cancer screening and the role of nursing: an integrative review
}

Barreras en la detección del cáncer de mama y el papel de la enfermería: una revisión integradora

\section{Tânia Silveira Lourenço', Edmundo Carvalho Mauad", René Aloisio da Costa Vieira'}

' Hospital de Câncer de Barretos, Departamento de Prevenção. Barretos-SP, Brasil.

Universidade Estadual Paulista "Júlio de Mesquita Filho", Faculdade de Medicina de Botucatu, Programa de Pós-Graduação em Pesquisa e Desenvolvimento - Biotecnologia Médica. Botucatu-SP, Brasil.

"Hospital de Câncer de Barretos, Departamento de Prevenção. Barretos-SP, Brasil.

\author{
Submissão: 09-01-2011 Aprovação: 31-05-2013
}

\section{RESUMO}

O rastreamento para o câncer de mama ou a realização regular do exame de mamografia é uma realidade em países desenvolvidos. A enfermagem atua de diferentes maneiras neste processo, tendo importante papel no enfrentamento das barreiras relacionadas com a não adesão ao rastreamento; porém, sua efetividade é pouco comprovada, havendo limitado número de estudos clínicos sobre o tema. Realizou-se revisão integrativa sobre o tema na PubMed e na literatura latino-americana LILACS, avaliando estudos clínicos controlados que comprovassem a efetividade das ações de enfermagem, utilizando as palavras-chave rastreamento para o câncer de mama, enfermagem e estudos clínicos. Na PubMed foram identificadas 110 publicações sobre o tema, das quais 18 estudos clínicos apresentando as ações de enfermagem. Não se identificou nenhum estudo na LILACS. A presente revisão mostrou a efetividade da enfermagem no contexto do rastreamento do câncer de mama, apresentando novas perspectivas de atuação profissional, dentro de um contexto multidisciplinar de qualificação de ações relacionadas à saúde da mulher.

Descritores: Neoplasias da Mama; Programas de Rastreamento; Papel do Profissional de Enfermagem; Enfermeira Oncológica; Ensaio Clínico.

\begin{abstract}
Mammographic breast cancer screening is a fact in developed countries. Nursing acts in different ways in this process, having an important role in the barriers related to non-adherence to screening, but its effectiveness is little proven, with limited number of clinical trials about this topic. An integrative review of the literature in PubMed and LILACS (Latin American Literature) was conducted, aiming to evaluate controlled clinical trials about nurse's role in breast cancer screening. It was used the key words breast cancer screening, clinical trials and nurse. From 110 publications in PubMed, 18 were clinical trials and showed the nurse's role in breast cancer screening. None publication was observed in LILACS database. This review shows the effectiveness of nursing in breast cancer screening, presenting new nurse's role in the multidisciplinary team that qualify actions related to women's health.
\end{abstract}

Key words: Breast Neoplasms; Mass Screening; Nurse's Role; Oncologic Nursing; Clinical Trial.

\section{RESUMEN}

La detección del cáncer de mama por mamografía es un hecho en los países desarrollados. Enfermería actúa de diferentes maneras en este proceso, asumiendo un papel importante en los obstáculos relacionados con la falta de adherencia a los exámenes. A pesar de su importancia, existe un número limitado de ensayos clínicos sobre este tema. Se realizó una revisión integrada de la literatura en PubMed y LILACS (Literatura Latinoamericana), evaluando ensayos clínicos sobre el papel de la enfermera en el rastreamento del cáncer de mama. Utilizó-se las palabras clave detección del cáncer de mama, enfermería y estudios clínicos. De 110 publicaciones en PubMed, 18 eran ensayos clínicos que mostraron el papel de la enfermera en la detección del cáncer de mama, pero ninguna publicación estaba presente en la base de datos LILACS. Esta revisión muestra la eficacia de la enfermería en la detección del cáncer de mama, presentando un nuevo papel de la enfermera en el equipo multidisciplinario y calificando las acciones relacionadas con la salud de la mujer.

Palabras clave: Neoplasias de la Mama; Tamizaje Masivo; Rol de la Enfermera; Enfermería Oncológica; Ensayo Clínico. 


\section{INTRODUÇÃO}

Estima-se que, por ano, ocorram mais de 1.050 .000 casos novos de câncer de mama em todo o mundo, sendo estimado para 2020, 15 milhões de casos novos anuais, dos quais $60 \%$ ocorrerão em países em desenvolvimento ${ }^{(1)}$. No Brasil, o câncer de mama representa o principal tipo de câncer na mulher, e o segundo tumor mais frequente na população feminina quer pela sua frequência, quer pela sua mortalidade ${ }^{(2)}$.

O prognóstico do câncer de mama é considerado bom. Verifica-se que a sobrevida nos países desenvolvidos é na ordem de $73 \%$ e nos países em desenvolvimento de $57 \%$. Nos EUA a elevação na incidência tem se associado à diminuição da mortalidade, observando-se uma sobrevida de $84.1 \%$ em 5 anos $^{(3)}$, fato este consequente aos avanços no tratamento e à realização de programas de rastreamento ${ }^{(1,3-4)}$. No Brasil, a elevação da incidência tem se associado à elevação na mortalidade ${ }^{(2)}$, e a sobrevida é de $67.8 \%$ aos $5 \operatorname{anos}^{(4)}$, decorrente do limitado números de mulheres diagnosticadas precocemente $^{(3,5-6)}$.

$\mathrm{Na}$ Europa, apesar de diferenças entre nações ${ }^{(7)}, \mathrm{o}$ rastreamento mamográfico é uma realidade, baseando-se em normatizações determinadas pela Rede Europeia de Câncer de Mama (Euporean Breast Cancer Network) ${ }^{(8)}$. Nos Estados Unidos, há elevadas taxas de adesão à mamografia ${ }^{(9)}$, havendo um programa nacional de acreditação de serviços diagnósticos (National Accreditation Program for Breast Centers) ${ }^{(10)}$. No Brasil, apesar do conhecimento de todos os processos relacionados ao rastreamento mamográfico ${ }^{(11)}$, há dificuldade na formalização de sistemas de referência efetivos, que atendam toda a população ${ }^{(12-13)}$, sob a forma de um rastreamento mamográfico organizado em mulheres assintomáticas ${ }^{(11)}$.

Há inúmeras razões relacionadas com a não realização do exame de mamografia, sendo sinteticamente divididas em barreiras relacionadas ao sistema de saúde, barreiras relacionadas à educação e barreiras relacionadas à atitude ${ }^{(14-16)}$. No Brasil, devido à ausência de rastreamento mamográfico nacional organizado, e à existência de experiências isoladas ${ }^{(17-19)}$, há limitados estudos clínicos relacionados ao papel da enfermagem no rastreamento mamográfico.

A enfermagem tem importante papel no rastreamento mamográfico organizado, sendo parte integrante da equipe multidisciplinar da Unidade da Mama (Breast Unit), devendo possuir qualificação específica para atuar no âmbito da comunicação, coordenação, manutenção dos dados ${ }^{(8,10)}$, educação e aconselhamento genético ${ }^{(10)}$. No nosso meio, é descrita sua ação na identificação da população alvo, treinamento profissional e em atividades que buscam a adesão das mulheres ao exame de mamografia ${ }^{(11)}$, porém há limitado número de estudos controlados relatando a efetividade da enfermeira neste contexto, fato que justifica uma revisão sobre o tema, objetivo deste trabalho.

\section{MÉTODO}

Foi realizado levantamento bibliográfico avaliando ensaios clínicos que avaliassem o papel da enfermagem em relação ao rastreamento mamográfico do câncer de mama. Inicialmente, visando contextualização, realizou-se levantamento de artigos relacionados à epidemiologia do câncer de mama, rastreamento mamográfico organizado e barreiras relacionadas a não adesão ao rastreamento. A revisão integrativa foi realizada mediante busca de artigos científicos indexados em base de dados internacional da U.S. National Libary of Medicine/ National Institutes of Health (PubMed; http://www.ncbi. nlm.nih.gov/pubmed) e na base de dados da literatura Latino-Americana (LILACS; http:// http://lilacs.bvsalud.org/).

Para os resultados apresentados, utilizou-se como filtro a presença de ensaios clínicos. Não houve limite na data inicial da publicação, porém utilizou-se o dia 31/12/2012, como limite máximo para publicação. Na PubMed foram utilizadas as palavras-chave "breast cancer screening e "nurse", seguido da procura de estudos clínicos (clinical trials). Inicialmente, foram identificados os títulos e lidos os resumos dos trabaIhos. Posteriormente, os trabalhos foram separados em função do conteúdo e pertinência. Na LILACS, baseado nos descritores da saúde (DeCS) foram utilizadas as palavras "neoplasias da mama", "enfermagem" e "ensaio clínico".

Os resumos que não apresentassem coerência com o tema foram excluídos. Dos resumos com coerência, os artigos foram avaliados, e na ausência de relação com o tema em questão foram excluídos, permanecendo no estudo apenas os estudos clínicos relacionados ao papel da enfermagem no rastreamento mamográfico.

\section{RESULTADOS}

Na PubMed, utilizando-se as palavras chave breast cancer screening e nurse, observou-se 1.463 publicações. Porém, ao separar por estudos clínicos, permaneceram 110 publicações, servindo como base inicial ao presente estudo. Na avaliação dos títulos e resumos, foram excluídos 88 artigos, visto que 55 relacionavam-se ao câncer, 8 ao diagnóstico, 7 ao risco de câncer, 5 à biologia molecular e 14 relacionados à enfermagem (8 relatam o conhecimento da enfermagem, 4 a educação em enfermagem e em 2 a enfermeira coletou os dados), restando 21 estudos para avaliação. Posteriormente foram excluídas 3 publicações, visto que a enfermeira fazia parte do processo de aconselhamento genético ${ }^{(20)}$ ou participou do exame clínico da mama ${ }^{(21-22)}$, porém sua atuação não foi avaliada nesses estudos. Das 18 publicações pertinentes à atuação da enfermagem no rastreamento do câncer de mama, os artigos foram separados frente ao papel da enfermagem no sistema de saúde $^{(23)}$, na educação ${ }^{(24-32)} \mathrm{e}$ na adesão ao rastreamento ${ }^{(33-40)}$.

Na base de dados do LILACS, utilizando-se os termos "neoplasia da mama" e "enfermagem", formam observadas 141 publicações, destas, 100 encontravam-se relacionadas a pacientes com câncer; 14 apresentavam temática relacionada com o rastreamento, não necessariamente implicando em ações de enfermagem; 9 focalizavam a formação ou conhecimento da enfermeira frente ao rastreamento; 7 relatavam avaliação diagnóstica em saúde; 3 relatavam a atuação da enfermeira no exame-clínico da mama; 3 utilizaram a enfermeira como sujeito da pesquisa; 3 relataram a consulta de enfermagem ( 1 no 
aconselhamento genético); e 2 eram revisões sobre ações de enfermagem no rastreamento. Porém, ao se avaliar "neoplasia da mama", "enfermagem" e "ensaio clínico", não se observou nenhuma publicação.

\section{DISCUSSÃO}

A prevenção secundária constitui o diagnóstico precoce do câncer. O câncer de mama pode ser detectado através de exames menos sofisticados, como é o caso do autoexame da mama (AEM) ou o exame clínico da mama (ECM), que pode ser realizado por profissionais capacitados e treinados. Estratégias avaliando o ECM são de difícil mensuração, visto a variedade entre observadores, bem como o fato de sua pouca sensibilidade $^{(41)}$, que varia de $57 \%$ a $83 \%{ }^{(42)}$.

Apesar de difundido entre as mulheres, a realização adequada do AEM ocorre em apenas uma parcela das mulheres. O AEM leva a mulher a uma avaliação médica, sendo observadas alterações benignas na ordem de 11,4\%; entretanto, a taxa de detecção de lesões malignas é baixa, fazendo parte integrante do processo de conscientização frente ao câncer de mama ${ }^{(42)}$. Da mesma forma, mulheres sintomáticas ou com nódulos palpáveis devem ser encorajadas a uma avaliação especializada.

Estudos prospectivos controlados, avaliando o papel do AEM, não conseguiram demonstrar a redução da mortalidade pelo câncer de mama ${ }^{(43)}$, e atualmente o AEM é sugerido no contexto do autoconhecimento do corpo, sendo desencorajado como estratégia de rastreamento do câncer de mama. Porém, devido a disparidades econômicas entre as nações, a Organização Mundial de Saúde (OMS) sugere que em países com recursos limitados, o AEM e o ECM devem ser valorizados como métodos de diagnóstico do câncer de mama, porém na presença de recursos econômicos, deve-se realizar o rastreamento mamográfico organizado em mulheres com idade superior aos 50 anos $^{(44)}$.

Segundo as recomendações da American Cancer Society (ACS), o exame clínico da mama deve ser iniciado aos 20 anos de idade, com intervalo trienal até os 30 anos, quando deve ser realizado anualmente. No Brasil, o Consenso de Controle do Câncer de Mama, principal documento do Programa Nacional de Controle do Câncer de Mama, preconiza o exame clínico da mama a partir dos 40 anos com periodicidade anu$\mathrm{al}^{(43,45)}$. A ACS, o Canadian Task Force e a Sociedade Brasileira de Mastologia recomendam o uso rotineiro da mamografia a mulheres com idade acima dos 40 anos. Por outro lado, a US Task Force e o Instituto Nacional do Câncer do Brasil sugerem a utilização da mamografia após os 50 anos para as mulheres assintomáticas ${ }^{(43)}$. A OMS concluiu através de estudo dos ensaios clínicos que é necessário ampliar o rastreamento entre a faixa etária de 40 a 69 anos, devendo ser o intervalo anual ou bianual(46).

O rastreamento mamográfico para o câncer de mama é a melhor metodologia de prevenção secundária a nível populacional, constituindo medida de intervenção, promovendo a detecção precoce na fase assintomática e implicando na redução substancial da morbimortalidade causada pelo diagnóstico tardio. Estudos prospectivos controlados demonstraram que o rastreamento mamográfico leva a uma maior taxa de detecção de lesões mamárias, associando-se a uma diminuição da mortalidade por câncer que chega a até $35 \%(43,46)$. A alteração da mortalidade ocorre, principalmente, em muIheres na faixa etária dos 50 a 69 anos, porém o benefício na faixa etária dos 40 a 49 anos também já foi provado(47).

\section{A. Barreiras relacionadas com a não adesão ao exame de mamografia}

O rastreamento mamográfico organizado é uma realidade na Europa ${ }^{(7)}$ e a realização regular do exame de mamografia é uma realidade nos Estados Unidos ${ }^{(9,48)}$. A enfermeira, no contexto de equipe multidisciplinar ${ }^{(8,10)}$, pode atuar desde a seleção de mulheres e o diagnóstico até o tratamento do câncer de mama, participando do gerenciamento, educação, desmistificação, acesso das mulheres, coleta de dados e apoio psicológico $^{(27)}$; porém, devido a diferenças econômicas e culturais $^{(9,48,49)}$, há diversas barreiras relacionadas ao rastreamento mamográfico ${ }^{(14-16)}$. Essas barreiras podem ser relacionadas ao sistema de saúde, à educação e à paciente (Figura I), sendo que a proporção varia em função das características culturais e socioeconômicas de cada país. Devido à complexidade do tema, será discutido cada fator em separado, avaliando o papel da enfermagem neste contexto.

Figura 1 - Barreiras relacionadas com a não adesão ao exame de mamografia. Modificado para países em desenvolvimento, a partir do modelo proposto por Rimer et $\mathrm{al}^{(14)}$.

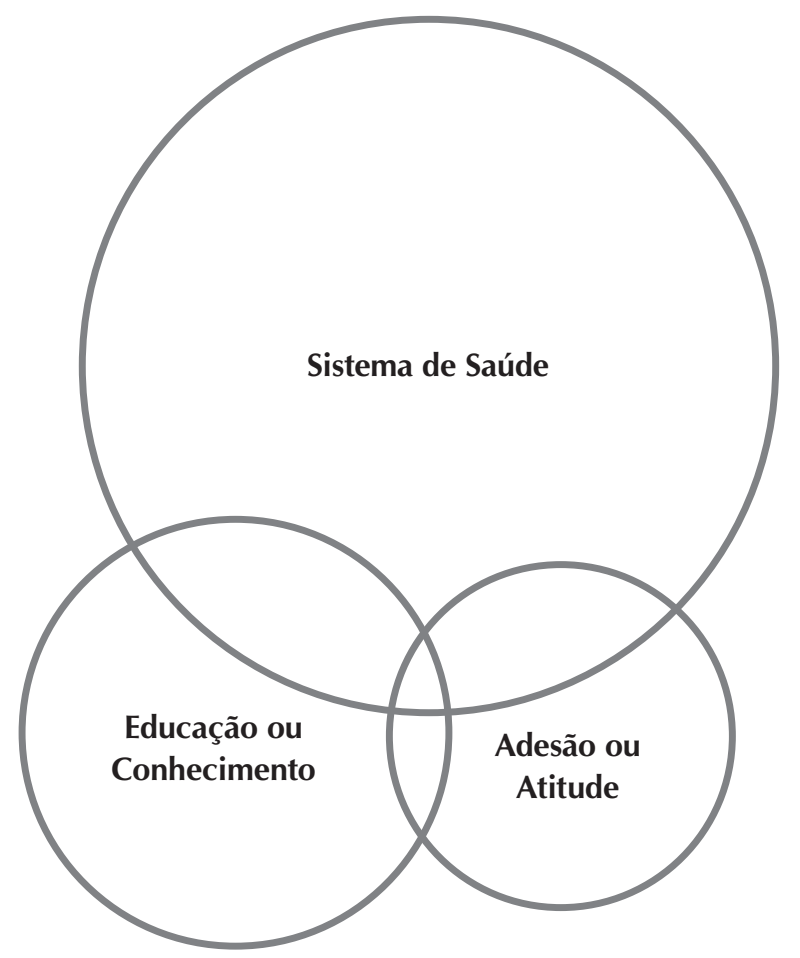

Barreiras relacionadas ao Sistema de Saúde

Nos EUA a taxa de realização de exame de mamografia é da ordem de $70 \%$ da população, sendo superior nas mulheres 
brancas $(72,1 \%)$ e escolaridade elevada $(80,1 \%)$, nascidas nos EUA $(71,6 \%)$ e portadoras de convênio médico $(73,6 \%)^{(48)}$. No Brasil, a taxa de mulheres que realizam mamografia na faixa dos 40 aos 69 anos é de 44,6\%, sendo inferior no Estado de Tocantins $(21,7 \%)$ e a maior taxa no Estado de São Paulo $(61,1 \%)$. Avaliando-se a taxa de mamografias realizadas apenas pelo Sistema Único de Saúde (SUS), nos estados anteriormente descritos, observa-se taxa de 6,9\% e 19,2\%, respectivamente $^{(50)}$, fato que denota um déficit de exames de mamografia em nível populacional, e uma limitação no sistema de saúde pública. Além disso, há demora no diagnóstico mamográfico, determinando baixos índices de tumores precoces ${ }^{(51)}$.

Dentre as barreiras relacionadas ao sistema de saúde para realização do exame temos: (1) acessibilidade aos serviços de saúde; (2) dificuldade de realização de exames de seguimento; (3) custo dos exames; (4) adesão médica insatisfatória, decorrente das limitações do sistema público de saúde ${ }^{(14-16)}$.

Países em desenvolvimento apresentam limites orçamentários na área de saúde pública. Os recursos são direcionados principalmente ao tratamento das doenças e são reduzidos os orçamentos direcionados à prevenção primária e secundária de doenças degenerativas e o câncer, determinando taxas elevadas de tumores avançados, por ocasião do diagnóstico. Para se obter a redução da mortalidade do câncer de mama, relacionada ao diagnóstico precoce pela mamografia, esta deve ser realizada em larga escala em nível populacional.

As barreiras econômicas são muitas vezes citadas na literatura, ocorrendo principalmente nas mulheres que não possuem convênio médico e possuem recursos limitados para pagamento do exame de mamografia, sendo este um dos principais fatores relacionados a não adesão aos exames de mamografia, principalmente em populações carentes ${ }^{(48)}$.

Estudo caso-controle avaliando a presença da enfermeira em unidades mamárias, observou uma elevação tanto na adesão ao exame de mamografia, como ao retorno bianual, nas unidades onde a enfermeira se encontra presente ${ }^{(23)}$, porém ao associar-se a mamografia ao exame físico realizado pelas enfermeiras, observou-se uma elevação do número de casos falso-positivos ${ }^{(22)}$.

\section{Barreiras relacionadas à educação ou conhecimento}

Em relação às barreiras relacionadas à educação ou conhecimento são citados: (1) determinantes culturais frente ao conceito de saúde; (2) características pessoais de educação, idade e sexo; (3) práticas estabelecidas de prioridades e projetos; (4) demora no rastreamento apropriado; (5) consumo de tempo; (6) características do indivíduo como etnia, idade, classe socioeconômica e estado educacional ${ }^{(14-16)}$.

No Brasil, nos últimos anos campanhas de televisão têm sido focadas no AEM, porém não há campanhas voltadas para a realização da mamografia, fato que determina um conhecimento limitado da população frente à problemática do câncer de mama. Os principais fatores considerados na não realização do exame de mamografia são escassez de equipamentos $(75,3 \%)$, custo do exame da mamografia $(65,7 \%)$, planos de saúde $(42,0 \%)$, resistência da mulher à realização do exame $(39,0 \%)^{(12)}$. Para a população os fatores relacionados a não adesão dos exames de mamografia para pacientes com convênio são a ausência de solicitação do exame pelo médico $(93,3 \%)$, e por parte do sistema público, ausência de solicitação do exame por parte do médico $(46,0 \%)$, ausência de motivação da mulher por considerar o exame desnecessário $(29,9 \%)$, e ausência de recurso financeiro $(9,0 \%)^{(13)}$.

Avaliando-se estudos controlados frente ao papel da enfermagem na educação, constatou-se 9 estudos relacionados à educação, 6 referem-se ao papel da educação no ECM e/ou $\mathrm{AEM}^{(24-26,29-30,32)} 1$ refere-se à educação da população frente à mamografia ${ }^{(31)}, 1$ avalia o impacto de ações de enfermagem na educação e elevação da adesão(23,27) e 1 refere-se à educação frente ao suporte ao diagnóstico ${ }^{(28)}$. Destes estudos, 6 foram randomizados ${ }^{(25-27,29-30,32)}$.

A atuação da enfermeira na educação frente ao ECM e/ou AEM é efetiva ${ }^{(24-26,29-30)}$ melhorando a sensibilidade na detecção de nódulos ${ }^{(24,26,30)}$, porém diferentes metodologias utilizadas de maneira isolada na educação não tiveram impacto na elevação da adesão ${ }^{(25,29-30,32)}$. Fato é que o ECM e/ou AEM eleva a taxa de exames falso-positivos ${ }^{(22)}$, sendo atualmente desencorajado em locais onde a mamografia encontra-se disponível.

Frente à mamografia, a maioria dos estudos mostra que as ações de enfermagem permitem uma elevação na educação e adesão na área onde essa atua ${ }^{(23,27)}$, e no estudo onde tal achado não se mostrou significante, o número de pacientes e o tempo de seguimento eram pequenos ${ }^{(31)}$. Após a realização do exame de mamografia, muitas mulheres são convocadas para novos exames diagnósticos, e nessa fase a enfermeira também colabora para o autocuidado das mulheres ${ }^{(20)}$.

\section{Barreiras relacionadas à adesão ou atitude}

Quanto às barreiras individuais, relacionadas à atitude das mulheres são citadas: (1) atitudes e conhecimentos frente ao câncer; (2) ausência de adesão às recomendações sugeridas pelo sistema de saúde; (3) desconforto gerado pelo exame; (4) medo do exame se mostrar positivo; (5) características do indivíduo como raça, idade, classe socioeconômica e escolaridade; (6) distância do local do exame; (7) meio de transporte para chegar até o local do exame ${ }^{(14-16)}$.

Em locais onde o exame de mamografia é disponível e a população tem conhecimento frente à importância do exame de mamografia, há uma parcela da população que não realiza a mamografia com regularidade ${ }^{(52-55)}$. Observa-se na literatura, artigos relatando a presença de conhecimento parcial e errôneo sobre a problemática do câncer de mama, tanto no que se refere à populações de risco, história familiar, ou diagnóstico precoce, quanto no que se refere à elevação das taxas de cura. Observa-se também que o medo do diagnóstico positivo, o medo do desconforto do exame, considerando-se a mamografia como desnecessária e diminuindo as taxas de adesão ${ }^{(52,55-56)}$. Há desconhecimento frente ao intervalo da realização do exame, determinando a não realização regular.

Em locais onde há disponibilidade de exames, recomenda-se a intensificação de intervenções de enfermagem em minorias, isto é, mulheres idosas, provenientes de minorias étnicas, com baixa escolaridade e pobres ${ }^{(16)}$. Em migrantes, o pequeno tempo de migração, não entendimento adequado da língua, 
ausência de emprego, custo do exame, limitação na disponibilidade de transporte, pobre motivação pessoal e limitações educacionais encontram-se relacionadas à baixa adesão ao exame de mamografia ${ }^{(54,56)} \mathrm{A}$ idade é o fator de risco mais importante para o câncer de mama, no entanto, a população idosa tem menor adesão à realização do exame de mamografia. Nas idosas, a limitação de recursos econômicos ${ }^{(57)}$ e a presença de doenças associadas conduzem a atenção à saúde em outra direção. Ao se considerar o grau de escolaridade, observou-se que a diminuição da escolaridade encontra-se relacionada à diminuição na adesão ao exame ${ }^{(48)}$. Por outro lado, o estado marital e a presença de filho(a)s levam a muIher a uma conscientização melhor frente à problemática da mama, elevando as taxas de adesão ${ }^{(52)}$.

No que se refere aos estudos controlados em enfermagem e à atitude da mulheres frente ao exame de mamografia, identificou-se 8 estudos $^{(33-37,39-40)}$, sendo 4 randomizados ${ }^{(36-38,40)}$. Frente à adesão, o uso de vídeos não surtiu efeito ${ }^{(35)}$, o uso de carta se mostrou efetivo ${ }^{(33,39-40)}$, porém a educação presencial por parte da enfermeira elevou a adesão, tanto de maneira significativa $^{(39)}$ como não significativa ${ }^{(38)}$. Do mesmo modo, a enfermeira como educadora ${ }^{(34)}$, atuando também na informação junto ao exame de mamografia ${ }^{(36)}$, eleva as taxas de adesão nos exames de seguimento.

\section{B. Ações da enfermagem no rastreamento do câncer de mama na literatura latino-americana}

No Brasil, não há rastreamento de base populacional organizado, observando-se relatos pontuais ${ }^{(17-19)}$, fato que dificulta a publicação de experiências neste contexto, bem como o delineamento de estudos clínicos de ações de enfermagem, comprovado pela ausência de ensaios clínicos publicados no LILACS. Apesar da importância do tema, estudos de revisão no $L / L A C S^{(58-59)}$ não conseguiram demonstrar a importância e a efetividade da enfermagem no contexto do rastreamento mamográfico, fato que justifica esta publicação, a qual mostra uma revisão integrativa sobre o tema, enfatizando a efetividade de ações demonstradas através de estudos clínicos.

Estudos não controlados descrevem que a enfermeira pode atuar no rastreamento, no âmbito do planejamento ${ }^{(19)}$, divulgação ${ }^{(17-19)}$, execução(19), adequação, manutenção e aprimoramento de processo ${ }^{(3,18)}$, como gestor(a) ${ }^{(3)}$ ou como educador(a) ${ }^{(59)}$ informando sobre a importância de adesão a recomendações de sociedades médicas ou órgãos de saúde, tanto na realização do $\mathrm{AEM}^{(17)}$ como da mamografia ${ }^{(18,60)} \mathrm{Ou}$ no conceito de "saúde mamária". Nesse contexto, a informação acerca do câncer de mama, idade para realização do exame de mamografia, formas de detecção do câncer e fatores de risco para o desenvolvimento da doença, como elementos da educação em saúde podem influenciar favoravelmente na adesão, determinando elevação no diagnóstico precoce.

Avaliando-se as experiências nacionais publicadas em rastreamento, há descrição do papel da enfermeira na coordenação de atividades junto à Estratégia de Saúde da Família, coordenação de atividades para identificação de mulheres não aderentes ao exame de mamografia, viabilizando a busca ativa de mulheres e auxiliando no fluxo de atendimento das pacientes que serão submetidas ao exame, exercendo atividade educativa frente à mamografia, ao ECM e/ou AEM, atuando nas barreiras relacionadas à educação e à adesão, além de auxiliar nas ações de gestão ${ }^{(18,19,55)}$. O rastreamento mamográfico aprimora o contexto de saúde da mulher, que associado ao conceito de atendimento multidisciplinar, abre novas perspectivas de ação da enfermagem junto ao contexto de saúde pública.

\section{CONCLUSÕES}

O rastreamento mamográfico é uma realidade cada vez mais presente no contexto de saúde da mulher e as ações de enfermagem encontram-se presentes em todo este processo, mostrando-se efetivas. Há diversos fatores ou barreiras que podem limitar a efetividade do rastreamento mamográfico, quer estejam relacionados ao sistema de saúde, a educação ou a adesão ao exame de mamografia, situações estas onde estudos clínicos controlados demonstraram a efetividade das ações de enfermagem. O conhecimento de todo este processo é de fundamental importância, pois abre novas perspectivas de atuação profissional, dentro de um contexto multidisciplinar, associado à qualificação da saúde da mulher.

\section{REFERÊNCIAS}

1. Parkin DM, Bray F, Ferlay J, Pisani P. Global cancer statistics, 2002.CA Cancer J Clin 2005;25(2):74-108.

2. Ministério da Saúde [homepage na internet]. Instituto Nacional de Câncer - INCA. A situação do câncer no Brasil [acesso em 07 dez 2008]. Disponível em: http://www. inca.gov.br/situacao/.

3. Vieira RAC. Sobrevida de pacientes portadoras de câncer de mama. Estudo comparativo entre pacientes de Hospital Oncológico no Brasil e população Americana. Botucatu. [Pós-doutorado] - Faculdade de Medicina da UNESP; 2012.

4. Berry DA, Cronin KA, Plevritis SK, Fryback DG, Clarke
$\mathrm{L}$, Zelen $\mathrm{M}$, et al. Effect of screening and adjuvant therapy on mortality from breast cancer. $\mathrm{N}$ Engl J Med 2005;353(17):1784-92.

5. Jemal A, Siegel R, Ward E, Hao Y, Xu J, Murray T, et al. Cancer statistics, 2008. CA Cancer J Clin 2008; 58(2):71-96.

6. Lee $\mathrm{BL}$, Kiedke $\mathrm{PE}$, Barrios $\mathrm{CH}$, Simon $\mathrm{SD}$, Finkelstein DM, Goss PE. Breast cancer in Brazil: present status and future goals. Lancet Oncol 2012;13(3):e95-102.

7. Taran FA, Eggemann H. Breast Units in Europe - Certification in 9 European countries 9 years after the European Society of Mastology position paper. Breast Care 2009;4(4):219-222. 
8. Perry N, Broeders M, Wolf C, Tornberg S, Holland R, Von Karsa L. European guidelines for quality assurance in breast cancer screening and diagnosis. Fourth editionsummary document. Ann Oncol 2008; 19(4):614-622.

9. Smith RA, Cokkinides V, Brawley OW. Cancer Screening in the United States, 2012. A review of current American Cancer Society guidelines and current issues in cancer screening. CA Cancer J Clin 2012; 62(2):129-42.

10. National Accreditation Program for Breast Centers [homepage na internet]. American College of Surgeons; 2013 [acesso em 20 abr 2013]. Disponível em: http:// www.napbc-breast.org/

11. Vieira RAC, Mauad EC, Mattheus AGZ, Mattos JSC, Haikel Júnior RL, Bauab SP. Rastreamento mamográfico: começomeio-fim. Rev Bras Mastologia 2010;20(2):92-97.

12. Godinho ER, Kock HA. O perfil da mulher que se submete a mamografia em Goiânia: uma contribuição a bases para um programa de detecção precoce do câncer de mama. Radiol Bras 2002;35(3):139-45.

13. Godinho ER, Koch HA. Rastreamento do câncer de mama: aspectos relacionados aos médicos. Radiol Bras 2004;37(2):91-99.

14. Rimer BK. Adherence to cancer screening. In: Reintgen DS, Clark RA, editores. Cancer Screening. St Louis: Mosby; 1996 . p. 261-276.

15. Womeodu RJ, Bailey JE. Barriers to cancer screening. Med Clin North Am 1996;80(1):115-33.

16. George SA. Barriers to breast cancer screening: an integrative review. Health Care for Women Int 2000; 21(1):53-65.

17. Caleffi M, Ribeiro RA, Bedin AJJ, Viegas-Butzke JM, Baldisserotto FD, Skonieski GP, et al. Adherence to a breast cancer screening program and its predictors in underserved women in Southern Brazil. Cancer Epidemiol Biomark Prev 2010;19(10):2673-9.

18. Mauad EC, Nicolau SM, Moreira LF, Haikel RLJ, Longatto-Filho A, Baracat EC. Adherence to cervical and breast cancer programs is crucial to improving screening performance. Rural Remote Health 2009; 9(3):1241.

19. Mauad EC, Silva TB, Haikel RLJ, Bauab S, Longatto-Filho A. Is community intervention in breast cancer screening in Brasil feasible? J Med Screen 2011;18(1):51.

20. Holloway S, Porteous M, Cetnarskyj R, Anderson E, Rush $R$, Fry $A$, et al. Patient satisfaction with two different models of cancer service in south-east Scotland. Br J Cancer 2004;90(3):582-589.

21. Holowaty $\mathrm{PH}$, Miller $\mathrm{AB}$, Baines $\mathrm{CJ}$, Risch $\mathrm{H}$. Canadian National Breast Screening Study: first screen results as predictors of future breast cancer risk. Cancer Epidemiol Biomark Prev 1993;2(11):11-19.

22. Chiarelli AM, Majpruz $V$, Brown $P$, Thériaul $M$, Shumak $\mathrm{R}$, Mai $\mathrm{V}$. The contribution of clinical breast examination to the accuracy of breast screening. J Natl Cancer Inst 2009;101(18):1236-1243.

23. Chiarelli AM, Majpruz V, Brown P, Theriault M, Edwards $\mathrm{S}$, Shumak $\mathrm{R}$, et al. Influence of Nurses on compliance with breast screening recommendations in an Organized Breast Screening Program. Cancer Epidemiol Biomarkers
Prev 2010;19(3):697-706.

24. Budakoglu II, Maral I, Ozdemir A, Bumin MA. The effectiveness of training for breast cancer and breast selfexamination inwomen aged 40 and over. J Cancer Educ 2007;22(2):108-11.

25. Carter AC, Feldman JG, Tiefer L, Hausdorff JK. Methods of motivating the practice of breast self-examination: a randomized trial. Prev Med 1985;14(5):555-72.

26. Crombie K, Hancock K, Chang E, Vardanega L, Wonghongkul T, Chanakok $\mathrm{A}$, et al. Breast screening education at Australian and Thai worksites: a comparison of program effectiveness. Contemp Nurse 2005; 19(1-2):181-96.

27. Jerome-D'Emilia B, Merwin E, Stern S. Feasibility of using technology to disseminate evidence to rural nurses and improve patient outcomes. J Contin Educ Nurs 2010;41(1):25-32

28. Liao MN, Chen PL, Chen MF, Chen SC, Chen YH. Supportive care for Taiwanese women with suspected breast cancer during the diagnostic period: effect on healthcare and support needs. Oncol Nurs Forum 2009;36(5):585-92.

29. Liu CY, Xia HO, Isaman DM, Deng W, Oakley D. Nursing clinical trial of breast self-examination education in China. Int Nurs Rev 2010;57(1):128-34.

30. Ortega-Altamirano D, López-Carrillo L, López-Cervantes M. Strategies for teaching self-examination of the breast to women in reproductive age. Salud Publica Mex 2000;42(1):17-25.

31. Secginli S, Nahcivan NO. The effectiveness of a nursedelivered breast health promotion program on breast cancer screening behaviours in non-adherent Turkish women: A randomized controlled trial. Int J Nurs Stud 2011;48(1):24-36.

32. Pisani P, Parkin DM, Ngelangel C, Esteban D, Gibson L, Munson $\mathrm{M}$, et al. Outcome of screening by clinical examination of the breast in a trial in the Philippines. Int J Cancer 2006;118:149-154.

33. Dalessandri KM, Cooper M, Rucker T. Effect of mammography outreach in women veterans. West J Med 1998;169(3):150-2.

34. Foley EC, D'Amico F, Merenstein JH. Five-year follow-up of a nurse-initiated intervention to improve mammography recommendation. J Am Board Fam Pract 1995;8(6):452-6.

35. Grindel CG, Brown L, Caplan L, Blumenthal D. The effect of breast cancer screening messages on knowledge, attitudes, perceived risk, and mammography screening of African American women in the rural South. Oncol Nurs Forum 2004;31(4):801-8

36. Lauver DR, Settersten L, Kane JH, Henriques JB. Tailored messages, external barriers, and women's utilization of professional breast cancer screening over time. Cancer 2003;97(11):2724-35.

37. Margolis KL Menart TC. A test of two interventions to improve compliance with scheduled mammography appointment. J Gen Intern Med 1996;11:539-541.

38. Margolis KL, Lurie N, McGovern PG, Tyrrell M, Slather JS. Increasing breast and cervical cancer screening in lowincome women. J Gen Inter Med 1998; 13:515-521. 
39. Sharp DJ, Peters TJ, Bartholomew J, Shaw A. Breast screening: a randomised controlled trial in UK general practice of three interventions designed to increase uptake. J Epidemiol Community Health 1996;50(1):72-6.

40. Sutton S, Bickler G, Sancho-Aldridge J, Saidi G. Prospective study of predictors of attendance for breast screening in innerLondon. J Epidemiol Community Health 1994;48(1):65-73.

41. Marinho LAB, Gurgel MSC, Cecatti JG, Osis MJD. Conhecimento, atitude e prática do auto-exame das mamas em centros de saúde. Rev Saúde Pública 2003;37(5):576-82.

42. Xavier NL, Ribeiro PLI, Menke CH, Cavalheiro JAC, Xavier MC. Exame clínico das mamas no Programa de Saúde da Família: experiência no litoral sul do Brasil. Rev Bras Mastol 2008;18(1): 12-7.

43. Thuler LC. Considerações sobre a prevenção do câncer de mama feminino. Rev Bras Cancerol 2003; 49(4):227-238.

44. Anderson BO, Braun S, Carlson RW, Gralow JR, Lagios $M D$, Lehman $C$, et al. Overview of breast health care guidelines for countries with limited resources. The Breast 2003;9(S2): 42-50.

45. Gebrim LH, Quadros L G. Rastreamento mamográfico no Brasil. Rev Bras Ginecol Obstet 2006;28(6):319-323.

46. Lee $\mathrm{CH}$. Screening mammography: proven benefit, continued controversy. Radiol Clin North Am 2002; 40(3):395-407.

47. Shapiro S, Strax P, Venet L. Periodic breast cancer screening in reducing mortality from breast cancer. JAMA 1971;215(11):1777-1783.

48. Ward E, Jemal A, Cokkinides V, Singh GK, Cardinez C, Ghafoor A, et al. Cancer disparities by race/ethnicity and socioeconomic status.CA Cancer J Clin 2004;54(2):78-93.

49. Anderson BO, Shyan R, Eniu A, Smith RA, Yip CH, Bese $N S$, et al. Breast cancer in limited-resource countries: an overview of the breast health initiative 2005 Guidelines. The breast 2006;12(S1):S3-S15).

50. Ministério de Planejamento, Orçamento e Gestão. Instituto Brasileiro de Geografia e Estatística [homepage na internet]. Pesquisa nacional por amostra de domicílios: acesso e utilização dos serviços de saúde 2003. [acesso em 15 jan 2007]. Disponível em: http://www.ibge.com. br/home/estatistica/populacao/trabalhoerendimento/ pnad2003/ saude/default.shtm.

51. Lee $B L$, Lieke PER, Barrios $\mathrm{CH}$, Simon SD, Finkelstein DM, Goss PE. Breast cancer in Brazil: present status and future goals. Lancet Oncol 2012;13:95-102.

52. Aro AR, de Koning HJ, Absetz P, Schreck M. Two distinct groups of non-attenders in an organized mammography screening program. Breast Cancer Res Treat 2001;70(2):145-153.

53. Lagerlund $M$, Hedin A, Sparén P, Thurfjell E, Lambe M. Attitudes, beliefs, and knowledge as predictors of nonattendance in a Swedish population-based mammography screening program. Prev Med 2000;31(4):417-28.

54. Lagerlund M, Maxwell AE, Bastani R, Thurfjell E, Ekbom A, Lambe M. Sociodemographic predictors of nonattendance at invitational mammography screening--a population-based register study (Sweden). Cancer Causes Control 2002;13(1):73-82.

55. Lourenço TS, Vieira RAC, Mauad EC, Silva TB, Costa AM, Peres SV. Barreiras relacionadas à adesão ao exame de mamografia em rastreamento mamográfico na DRS-5 do estado de São Paulo. Rev Bras Mastol 2009;19(1):2-9.

56. Remennick L. The challenge of early breast cancer detection among immigrant and minority women in multicultural societies. Breast J 2006;12 (1):S103-110.

57. Kelaher M, Stellman JM. The impact of medicare funding on the use of mammography among older women: implications for improving access to screening. Prev Med 2000;31(6):658-664.

58. Fonseca ATL. Atuação da enfermagem na detecção precoce do cancer de mama. Rio de Janeiro. Monografia [Especialização em oncologia] - Instituto Nacional do Câncer; 2005.

59. Zapponi ALB, Tocantins FR, Vargens OMC. A detecção precoce do câncer no contexto brasileiro. Rev Enf UERJ 2012;20(3):386-90.

60. Soyer MT, Ciceklioglu M, Ceber E. Breast cancer awareness and practive of breast self examination among primary health care nurses: influencing factors and effects of an in-service education. J Clin Nurs 2007; 16(4):707-15. 